\title{
Finite element formulations for large strain anisotropic material with inextensible fibers
}

\author{
P. Wriggers ${ }^{1 *}$, J. Schröder ${ }^{2}$ and F. Auricchio ${ }^{3}$
}

\section{${ }^{*}$ Correspondence:}

wriggers@ikm.uni-hannover.de 'Institute for Continuum

Mechanics, Leibniz Universität Hannover, Hanover, Germany Full list of author information is available at the end of the article

\section{Springer Open}

\begin{abstract}
Anisotropic material with inextensible fibers introduce constraints in the mathematical formulations. This is always the case when fibers with high stiffness in a certain direction are present and a relatively weak matrix material is supporting these fibers. In numerical solution methods like the finite element method the presence of constraints - in this case associated to a possible fiber inextensibility compared to a matrix-lead to so called locking-phenomena. This can be overcome by special interpolation schemes as has been discussed extensively for volume constraints like incompressibility as well as contact constraints. For anisotropic material behaviour the most severe case is related to inextensible fibers. In this paper a mixed method is developed that can handle anisotropic materials with inextensible fibers that can be relaxed to extensible fiber behaviour. For this purpose a classical ansatz, known from the modeling of volume constraint is adopted leading stable elements that can be used in the finite strain regime.
\end{abstract}

Keywords: Anisotropic material, Finite element analysis, Mixed methods, Constraints

\section{Background}

Many different approaches were developed over the last decade to formulate finite elements for anisotropic material with inextensible fibers. The problem is the high stiffness ratio between fiber and matrix material with the limit case of inextensible fibers where this ratio tends to infinity. This is physically related to the exact fulfilment of the kinematic constraint associated with the inextensibility of fibers in certain directions.

Generally the method of Lagrange multipliers provides a possibility to fulfil such constraints for small and finite deformations. In this paper the Lagrange multiplier approach is employed to model anisotropic material behaviour at finite strains. Furthermore a relaxed version, i.e., the perturbed Lagrangian formulation, is used to model extensible fibers as well. Boundary value problems that incorporate extreme constraints cannot be solved using the finite element method with standard displacement interpolations. This leads to well known locking phenomena.

The main source of locking problems is that the mathematical formulation has to deal with constraints or is set up such that constraints are fulfilled approximately, like in penalty or other related methods. These problems are well-analyzed for geometrically linear prob-

(c) The Author(s) 2016. This article is distributed under the terms of the Creative Commons Attribution 4.0 International License (http://creativecommons.org/licenses/by/4.0/), which permits unrestricted use, distribution, and reproduction in any medium, provided you give appropriate credit to the original author(s) and the source, provide a link to the Creative Commons license, and indicate if changes were made. 
lems in the case of volume constraints, see e.g. $[4,13,28,30]$. They were investigated in the mathematical community quite early, see $[3,7]$, and are now well understood leading to the Babuska-Brezzi (BB) condition. It can be employed to investigate the stability behaviour of mixed finite elements in the linear range. Within nonlinear problems the $\mathrm{BB}$ condition can only be used at certain stages of the analysis, see e.g. [9].

Different strategies were pursued in computational mechanics over the last years in order to circumvent locking effects. It became evident that element ansatz functions that interpolate the deformation or displacement field within an element with first order shape functions (bi- or tri-linear interpolation) do not converge properly when applied to problems with constraints like incompressibility or distinct anisotropic material behaviour. Thus different variational formulations were explored in order to construct finite elements that can be used for problems with constraints. Approaches include reduced integration and stabilization, see e.g. [31] for the linear case. Many variants can be found in the literature. It was shown that the reduced integration has to be used together with stabilization and can be extended to nonlinear problems, see e.g. $[6,17]$ leading to elements that are in general locking free for incompressibie deformations. Additionally these elements are very efficient due to reduced integration. However stabilized elements rely on artificial stabilization parameters and thus the numerical solution can depend on theses parameters in certain cases.

Formulations, based on the mixed variational principle of Hu-Washizu, were developed, e.g. see Simo and co-workers who introduced the enhanced strain elements first for the geometrically linear, e.g. see [24] and then for large deformations, [22,23]. However, these elements depict non-physical instabilities at certain deformation states.

Other mixed finite element formulations, that are stable, perform well in the framework of small deformations and isotropy, e.g. see $[5,8]$. Extensions to problems undergoing finite deformations are discussed in $[1,2]$ for the case of incompressibility. For finite strain anisotropic material behavior it is even more complex to find good finite element formulations. Many classical approaches that were designed for fiber-reinforced materials depict non-physical behavior, see e.g. [12,27]. Discussions related to the correct formulations of the mathematical model for anisotropic behaviour can be found in e.g. [11,18]. These authors state that all fiber-related terms have to be provided in the energy by the complete deformation tensor and not by its isochoric part.

Reduced integration schemes using a special stabilization have been successfully applied to the simulation of composite reinforced material, see Hamila and Boisse [10]. Also special interpolations eliminated locking behaviour for composite materials, see ten Thjie and Akkerman [26]. Still many researchers use Hu-Washizu-based displacement, dilatation and pressure formulations, early introduced for incompressible materials by [25], for nearly incompressible materials with highly stiff fibers (like in arterial walls), see [29] and the references therein. However for strongly anisotropic material with inextensible fibers these approaches have limited performance, especially at finite strains.

A new formulation was presented in [21] who introduced a novel finite element formulation that is developed especially for anisotropic materials, based on isotropic tensor functions as discussed in $[19,20]$. There the constraints, associated with the anisotropy, are controlled by an additional deformation measure. A second-order tensorial Lagrange-multiplier was introduced via a discontinous ansatz. This approach offers the opportunity to reduce the interpolation order of the anisotropic part and 
thus is able to relax the constraints due to anisotropy. This formulation leads to a stable methods for the solution of problems with anisotropic materials undergoing large strains.

In this paper a different approach is followed. Here the constraint of inextensibility in fiber directions is formulated as a constraint and also as a limiting case. For this purpose a constraint equation is introduced within a Lagrange multiplier scheme. This allows to select ansatz functions as well for the displacement field in fiber direction as for the fiber forces. Additionally a perturbed Lagrangian formulation is introduced to relax the constraint condition and to be able to introduce real fiber stiffnesses. Since it can happen that fibers buckle locally when subjected to a compressive force a special form of the constraint is introduced that acts only for tension states. Furthermore this formulation can be used to enforce strain states in fiber direction that can be associated with e.g. muscle contractions in biomechanics applications or specific piezoelectric effects in fibers.

The performance of the developed element formulations is compared to existing formulations using benchmark problems. All numerical results were obtained with the AceGen/AceFEM system developed in [14-16].

\section{Anisotropic material with inextensible fibers behaviour at large strain}

In this section a summary of the continuum mechanics background is provided for the formulation of problems exhibiting anisotropic response in finite elasticity. The formulation is reduced to the necessary equations that are needed to formulate the problem in AceGen. This omits many derivations since automatic differentiation is used. All formulations are presented with respect to the initial configuration. The formulation accounts for transversely isotropic material behaviour by using a mixed approach. It is assumed that the material is not extendable in the given fiber direction $\mathbf{a}$.

\section{Continuum mechanics}

Let us introduce the deformation $\varphi(X, t)$ which maps points of the initial configuration to the current or deformed configuration. This deformation can be computed using the coordinates of the initial configuration and the displacement field: $\varphi(\mathbf{X}, t)=\mathbf{X}+\mathbf{u}(\mathbf{X}, t)$. Using this deformation map, the deformation gradient can be computed as

$$
\mathbf{F}=\operatorname{Grad} \varphi(\mathbf{X}, t)=\operatorname{Grad}(\mathbf{X}+\mathbf{u}(\mathbf{X}, t))=\mathbf{1}+\mathbf{H},
$$

where $\mathbf{H}=\operatorname{Grad} \mathbf{u}(\mathbf{X}, t)$. Note that the volume change $J$ is defined as the determinant of the deformation gradient: $J=\operatorname{det} \mathbf{F}$.

Based on the deformation gradient the Cauchy-Green tensor can be formulated as

$$
\mathbf{C}=\mathbf{F}^{T} \mathbf{F} .
$$

Based on these kinematical quantities one can formulate a strain energy function for hyperelastic materials. The following isotropic strain energy function $W^{\text {iso }}$ can be used to describe the behaviour of the isotropic part of the material:

$$
W^{i s o}(\mathbf{u})=\frac{\mu}{2}(\operatorname{tr} \mathbf{C}-3-2 \log J)+\frac{\lambda}{4}\left(J^{2}-1-2 \log J\right),
$$

where $\mu$ and $\lambda$ are the Lame constants, see e.g. [28]. Any other strain energy function that describes hyperelastic material behaviour can be selected as well. 


\section{Kinematical anisotropic constraint}

The enforcement of the constraint that ensures that the material does not extend in the direction a leads to the following condition

$$
\mathbf{a} \cdot \mathbf{E} \mathbf{a}=0,
$$

where $\mathbf{E}$ is the Green-Lagrangian strain tensor

$$
\mathbf{E}=\frac{1}{2}\left(\mathbf{F}^{T} \mathbf{F}-\mathbf{1}\right)=\frac{1}{2}(\mathbf{C}-\mathbf{1}) .
$$

Since it is simpler to work with the right Cauchy Green tensor $\mathbf{C}=\mathbf{F}^{T} \mathbf{F}$ this constraint can be written as

$$
2 \mathbf{a} \cdot \mathbf{E} \mathbf{a}=\mathbf{a} \cdot(\mathbf{C}-\mathbf{1}) \mathbf{a}=\mathbf{a} \cdot \mathbf{C} \mathbf{a}-1 \text { for }\|\mathbf{a}\|=1
$$

Furthermore we can write, by substituting the structural tensor $\mathbf{M}$,

$$
\mathbf{a} \cdot \mathbf{C} \mathbf{a}=\mathbf{C} \cdot \mathbf{M}=\operatorname{tr}[\mathbf{C M}] \text { with } \mathbf{M}=\mathbf{a} \otimes \mathbf{a} .
$$

It is easy to show that $\operatorname{tr}[\mathbf{C M}]$ yields the stretch in direction of $\mathbf{a}$. Thus

$$
\lambda_{c}^{2}=\operatorname{tr}[\mathbf{C M}]
$$

which in case of a fiber constraint in the direction of a leads to $\lambda_{c}^{2}=1$.

\section{Lagrange multiplier formulation}

Based on these kinematical relations different constraints and associated forms of a Lagrange multiplier approach can be formulated:

- One constraint. The Lagrange multiplier term related to the constraint of a material that is not extendable in the direction a yields with (7)

$$
W^{t i L}\left(\mathbf{C}, \sigma_{c}\right)=\sigma_{c}(\operatorname{tr}[\mathbf{C M}]-1)
$$

where $\sigma_{c}$ is the Lagrangian multiplier that physically represents the fiber stress related to the constraint.

- Several constraints. For more than one constraint direction one can introduce $n_{c}$ additional directional unit vectors $\mathbf{a}_{i}$ and associated structural tensors $\mathbf{M}_{i}$ and reformulate (9)

$$
W^{t i L}\left(\mathbf{C}, \sigma_{c i}\right)=\sum_{i=1}^{n_{c}} \sigma_{c i}\left(\operatorname{tr}\left[\mathbf{C M}_{i}\right]-1\right)
$$

- Constraints for tension only. In case that the response of the fiber system only occurs in tension states (9) can be re-written by using the Macauley bracket: $\langle x\rangle=\frac{1}{2}(x+\|x\|)$. This choice yields

$$
W^{t i L}\left(\mathbf{C}, \sigma_{c}\right)=\sigma_{c}\langle\operatorname{tr}[\mathbf{C M}]-1\rangle^{\alpha}
$$

where $\alpha$ is a positive integer that can be selected in the range $(1, \ldots, 4)$.

- Constraints for a given stretch. If a stretch $\bar{\lambda}_{c}$ is prescribed in a certain direction a, then one can formulate, using (8), the constraint

$$
W^{t i L}\left(\mathbf{C}, \sigma_{c}\right)=\sigma_{c}\left(\operatorname{tr}[\mathbf{C M}]-\bar{\lambda}_{c}^{2}\right) .
$$


Now one of the four variants of $W^{t i L}\left(\mathbf{C}, \sigma_{c}\right)$, discussed above, can be used to formulate the final form of the strain energy

$$
W(\mathbf{C}(\mathbf{u}), \sigma)=W^{i s o}(\mathbf{C}(\mathbf{u}))+W^{t i L}\left(\mathbf{C}(\mathbf{u}), \sigma_{c}\right) .
$$

All of the additions $W^{t i L}\left(\mathbf{C}, \sigma_{c}\right)$ to the strain energy (3) lead to a pure mixed form since unknowns are the displacement field $\mathbf{u}$ and the fiber stresses $\sigma_{c}$.

\section{Perturbed Lagrangian formulation}

Additionally there is the possibility to use a so called perturbed Lagrangian formulation which can be stated as follows

$$
W(\mathbf{C}, \sigma)=W^{i s o}(\mathbf{C})+W^{t i p L}(\mathbf{C}, \sigma) .
$$

with

$$
W^{t i p L}\left(\mathbf{C}, \sigma_{c}\right)=\sigma_{c}(\operatorname{tr}[\mathbf{C M}]-1)-\frac{1}{2 C_{c}} \sigma_{c}^{2}
$$

here again $C_{c}$ is a penalty parameter. For $C_{c} \rightarrow \infty$ (15) reduces to (13). The perturbed Lagrangian formulation leads in the continuous version to a penalty method, but for different ansatz spaces for $\sigma_{C}$ and the displacement field $\mathbf{u}$ it can lead to a different finite element scheme.

The perturbed Lagrangian formulation can also be used to introduce a fiber stiffness that is related to the physical behaviour of the fiber. In that case $C_{c}$ has a physical meaning.

\section{Penalty formulation}

Penalty methods provide a formulation that can approximate constraint equations by introducing a penalty term related to the constraint. The associated formulations includes the constraint (8) in the strain energy as follows

$$
W^{p}(\mathbf{C})=W^{i s o}(\mathbf{C})+W^{p e n}(\mathbf{C}) \text {. }
$$

with

$$
W^{p e n}(\mathbf{C})=\frac{C_{c}}{2}(\operatorname{tr}[\mathbf{C M}]-1)^{2}
$$

here $C_{c}$ is a penalty parameter. For $C_{c} \rightarrow \infty$ (15) it can be shown that the constraint is fulfilled exactly. ${ }^{1}$ The penalty formulation can also be used to introduce a certain fiber stiffness that is related to the physical behaviour of the fiber. In that case $C_{c}$ has a physical meaning.

\section{Mixed element formulation}

For the mixed interpolation tetrahedral and hexahedral elements are selected and compared. For both element formulations a quadratic interpolation for the displacement field $\mathbf{u}$ and a linear interpolation for the mixed variable $\sigma_{c}$ is selected. This choice is motivated by the classical mixed formulation for the incompressibility constraint. For anisotropic material with inextensive fiberss the variable $\sigma_{c}$ is the stress component related to the constraint, e.g. the stress in direction of $\mathbf{a}$.

${ }^{1}$ It is well known that ill-conditioning can occur when a large penalty parameter $C_{c}$ is selected. Thus in practise the penalty formulation is only able to approximately enforce the constraint condition (8). 
Note that in the mixed form for the incompressibility with the constraint $(J-1)$, that is related to the determinant of $\mathbf{F}$, a cubic function of the components of the deformation gradient describes this constraint. In the case of the constraint (9) for anisotropic materials this function is only a quadratic form of the components of the deformation gradient. Thus it is not obvious that the same choice for the interpolation of $\sigma_{c}$ will be sufficient. $^{2}$

For the formulation of the mixed finite element we start from Eq. (9). Thus one has to compute the structural tensor $\mathbf{M}$ that depends on the vector a providing the direction of anisotropy. Vector $\mathbf{a}$ is defined as a unit vector

$$
\mathbf{a}=\left\{a_{x}, a_{y}, a_{z}\right\} / \sqrt{a_{x}^{2}+a_{y}^{2}+a_{z}^{2}}
$$

Now ansatz functions for the displacement field and the Lagrangian multiplier (fiber stress) $\sigma_{c}$ have to be formulated. The quadratic shape functions that approximate the displacement field

$$
\mathbf{u}_{e}=\sum_{I=1}^{n_{u}} N_{I}(\xi, \eta, \zeta) \mathbf{u}_{I}
$$

are given below

- for a tetrahedron with 10 nodes $\left(n_{u}=10\right)$

$$
\begin{aligned}
& N_{1}=(2 \xi-1) \xi, \quad N_{2}=(2 \eta-1) \eta, N_{3}=(2 \zeta-1) \zeta, N_{4}=(2 \kappa-1) \kappa, \\
& N_{5}=4 \xi \eta, N_{6}=4 \eta \zeta, N_{7}=4 \zeta \xi, N_{8}=4 \xi \kappa, N_{9}=4 \eta \kappa, N_{10}=4 \zeta \kappa,
\end{aligned}
$$

with $\kappa=1-\xi-\eta-\zeta$ and

- a hexahedron with 27 nodes $\left(n_{u}=27\right)$

$$
N_{I}(\xi, \eta, \zeta)=N_{I}(\xi) N_{I}(\eta) N_{I}(\zeta)
$$

with $I=1, \ldots, 27 . N_{I}(s)$ is given for the vertex nodes by

$$
N_{I}(s)=\frac{1}{2}\left(1-s_{I}\right)[s(s-1)]+\frac{1}{2}\left(1+s_{I}\right)[s(s+1)]
$$

for $s$ being either $\xi, \eta$ or $\zeta$. Here $s_{I}$ is related to a specific coordinate of a vertex node of the hexahedron in the space of the reference coordinates $(\xi, \eta, \zeta)$ with $\xi_{I}=\{-1,+1\}$, $\eta_{I}=\{-1,+1\}$ and $\zeta_{I}=\{-1,+1\}$, see Fig. 1 . For the mid nodes the shape function $N_{I}(s)$ are given by

$$
N_{I}(s)=\left(1-s^{2}\right)
$$

with $\xi_{I}=0, \eta_{I}=0$ and $\zeta_{I}=0$.

Furthermore, the linear shape functions for the interpolation of the Lagrange multiplier $\sigma_{c}$ are defined for the tetrahedron with respect to the four edge nodes $\left(n_{\sigma}=4\right)$

$$
N_{\sigma 1}=\xi, \quad N_{\sigma 2}=\eta, \quad N_{\sigma 3}=\zeta, \quad N_{\sigma 4}=\kappa
$$

and for the hexahedron with respect ot the eight edge nodes $\left(n_{\sigma}=8\right.$ and $\left.K=1, \ldots, 8\right)$ as

$$
N_{\sigma K}=\frac{1}{8}\left(1+\xi \xi_{K}\right)\left(1+\eta \eta_{K}\right)\left(1+\zeta \zeta_{K}\right)
$$

\footnotetext{
${ }^{2}$ In the linear case both conditions, while being different, yield a linear dependence on the components of the displacement gradient. Thus there the choice of using the same ansatz function for the pressure (incompressibility) and the fiber stress (anisotropy) is justified.
} 


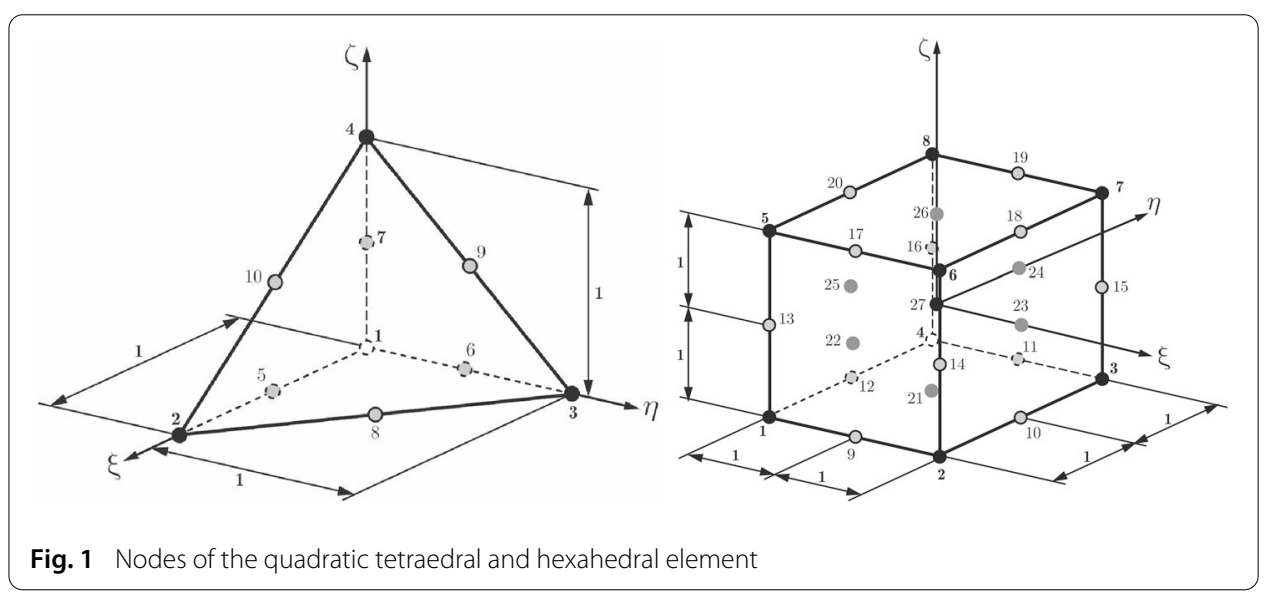

these will be used to interpolate the Lagrange multiplier (fiber stress) $\sigma_{c}$ related to the constraint within the element

$$
\sigma_{c}=\sum_{K=1}^{n_{\sigma}} N_{\sigma K}(\xi, \eta, \zeta) \sigma_{K} .
$$

Furthermore we need to define the coordinates within the finite element to formulate the isoparametric mapping. With $\zeta=(\xi, \eta, \zeta)$ it follows

$$
\mathbf{X}_{e}=\sum_{J=1}^{n_{u}} N_{J}(\zeta) \mathbf{X}_{J}
$$

Based on this ansatz functions the deformation gradient within an element $e$ is computed via

$$
\mathbf{F}_{e}=\mathbf{1}+\operatorname{Grad} \mathbf{u}_{e}=\sum_{I=1}^{n_{u}} \mathbf{u}_{I} \otimes \mathbf{J}_{e}^{-T} \nabla_{\zeta} N_{I}
$$

with the Jacobian of the isoparametric map

$$
\mathbf{J}_{e}=\sum_{I=1}^{n_{u}} \mathbf{X}_{I} \otimes \nabla_{\zeta} N_{I} .
$$

Now the Jacobian $J_{e}$ of the deformation gradient $\mathbf{F}_{e}$ is obtained within the element by $J_{e}=\operatorname{det} \mathbf{F}_{e}$. Furthermore the Cauchy-Green tensor $\mathbf{C}_{e}$ and the trace of $\mathbf{C}_{e} \mathbf{M}$ can then be computed at the element level. The latter quantity is needed to formulate the constraint (7).

In this contribution we will employ the tool AceGen to produce the finite element code. With all these kinematic quantities, one of the above strain energies, e.g. in (14) can be formulated. This is sufficient when AceGen is used to derive the element residual vector and the tangent matrix. The essential part of the AceGen code, related to the perturbed Lagrangian formulation is shown in Fig. 2.

\section{Examples}

Several numerical examples are considered to show the performance of the new formulation for different loading cases. In these examples the following discretization schemes are compared: 


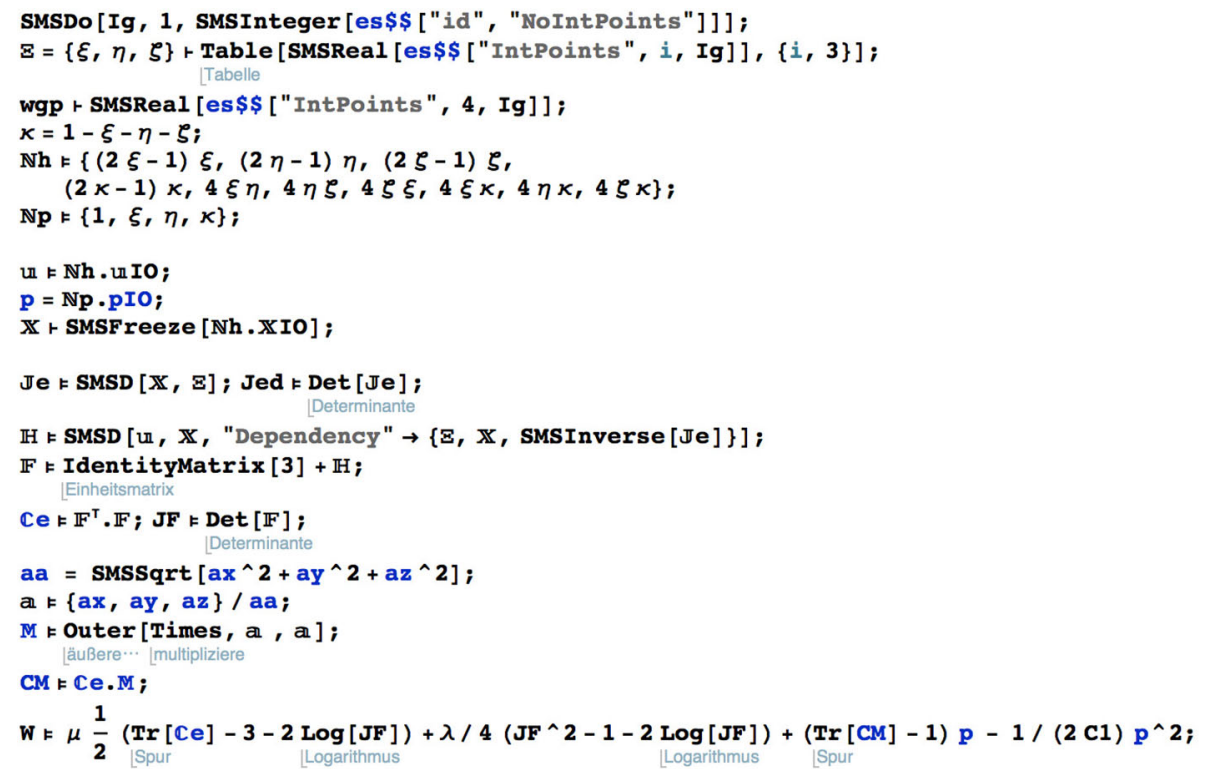

Fig. 2 Part of the AceGen code for the mixed element based on a perturbed Lagrangian formulation for transverely anisotropic material

- Tetrahedral elements for the constraint formulation (9), (10), (11) and (12) with quadratic ansatz functions (21) for the deformations and linear ansatz, see (23), for the Lagrangian multiplier $\sigma_{c}$. These elements are labeled T2-A1 in the following.

- Tetrahedral elements for the perturbed Lagrangian formulation (15) with quadratic ansatz functions (20) for the deformations and linear ansatz, see (22), for the Lagrangian multiplier $\sigma_{c}$. These elements are labeled T2-A1-P in the following.

- Hexahedral elements for the constraint formulation (9), (10), (11) and (12) with quadratic ansatz functions (20) for the deformations and linear ansatz, see (22), for the Lagrangian multiplier $\sigma_{c}$. These elements are labeled H2-A1 in the following.

- Hexahedral elements for the perturbed Lagragngian formulation (15) with quadratic ansatz functions (21) for the deformations and linear ansatz, see (23), for the Lagrangian multiplier $\sigma_{c}$. These elements are labeled H2-A1-P in the following.

For comparison reasons standard displacement elements were formulated as well as elements based on the penalty method (16). These elements are

- Tetrahedral elements based on the quadratic ansatz functions (20) for the deformations. These elements are labeled T2, and the associated penalty ones T2-P.

- Hexahedral elements based on the quadratic ansatz functions (21) for the deformations. These elements are labeled $\mathrm{H} 2$, and the associated penalty ones H2-P.

All examples are subjected to loads that lead to finite deformation strain states.

\section{Cook's membrane problem}

An example that will show a clear anisotropic response is the Cook's membrane problem of a tapered cantilever beam, clamped at the left end. The structure is loaded at the right end by a constant vertical load, as depicted in Fig. 3. 


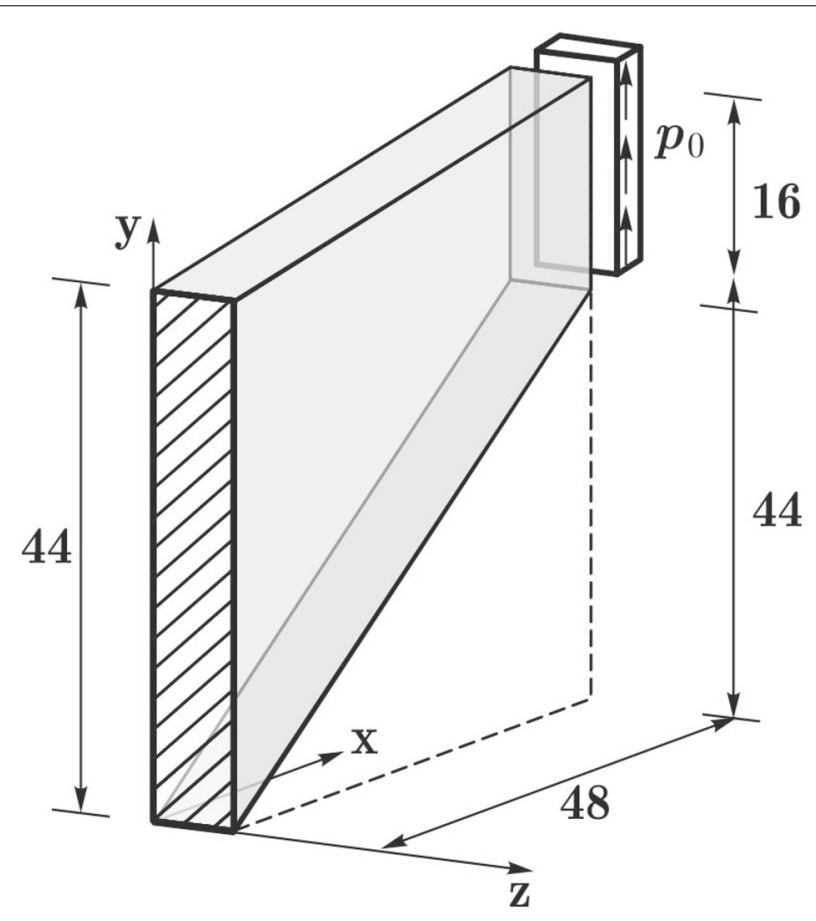

Fig. 3 Initial configuration of the cantilever beam

The selected dates for the Lame constants are $\mu=500$ and $\lambda=1000$. The direction of anisotropy is given by $\mathbf{a}=\frac{1}{\sqrt{3}}\{1,1,1\}$. In order to clamp the cantilever beam at its left end all displacements at $X=0$ were set to zero in $x$-, $y$ - and $z$-direction. The total distributed load is $p_{0}=250$. It was applied in different loading steps, as will be discussed later.

Different mesh densities where used to compute the solution, see Fig. 4 for the tetrahedra and Fig. 5 for the hexahedra. The mesh sequence is selected such that the finer meshes are included in the coarser meshes. This enables convergence studies that will depict differences of the formulations. The number $N$ denotes the mesh divison, see Table 1.

In a first computation a mesh with $N=16$ was used to obtain the load displacement curve for Cook's membrane problem. The element used for this simulation was the

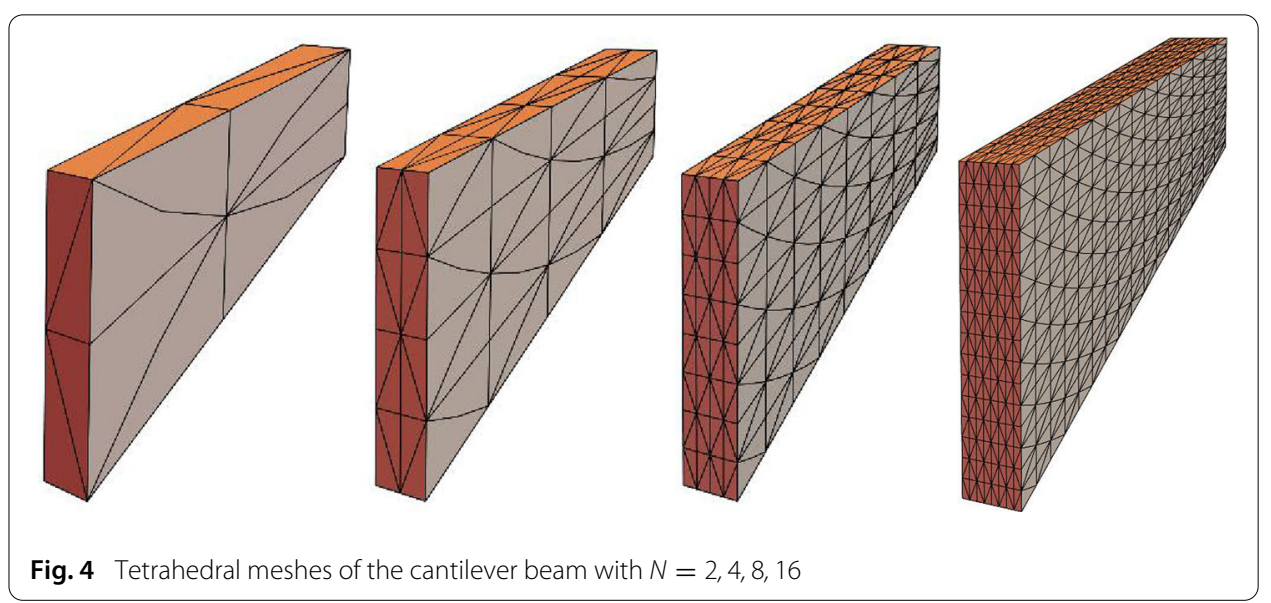




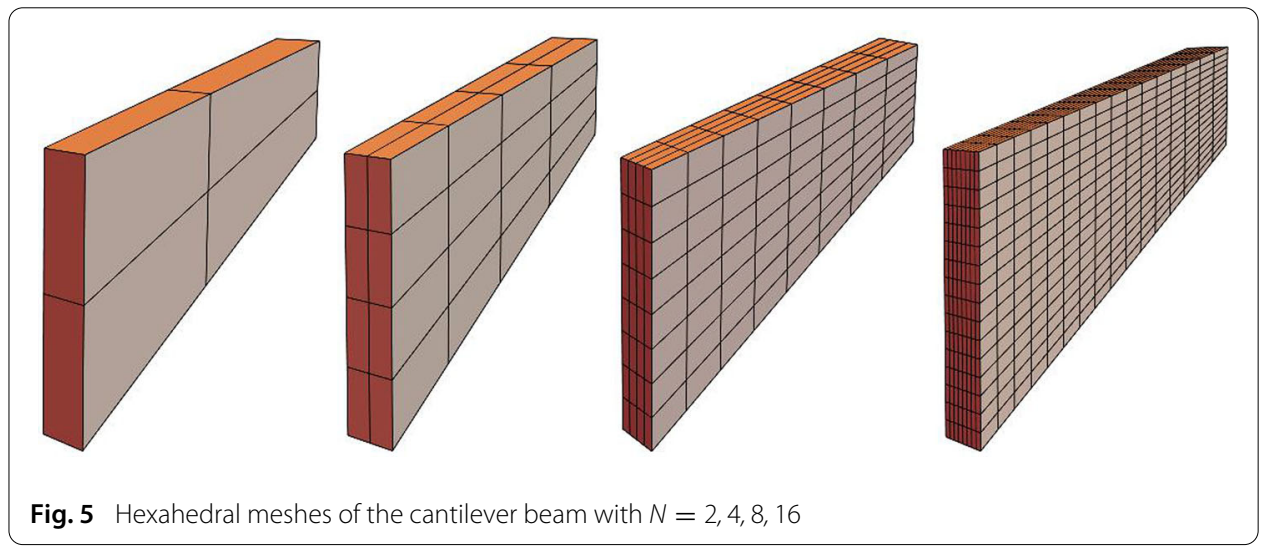

\section{Table 1 Mesh density}

\begin{tabular}{ll}
\hline $\boldsymbol{N}$ & Mesh division \\
\hline 2 & $2 \times 2 \times 1$ \\
4 & $4 \times 4 \times 2$ \\
8 & $8 \times 8 \times 4$ \\
16 & $16 \times 16 \times 8$ \\
\hline
\end{tabular}

H2-A1-P formulation. The load was applied in 10 even load increments $\lambda$ with $\Delta \lambda=0.25$. The parameter for the perturbed formulation was selected as $C_{c}=10^{6}$.

For the computation of the load displacement curve the vertical displacement of the mid node $(X, Y, Z)=(48,52,5)$ of the plane at the right end of the cantilever beam is chosen which is related to the response in the direction of the load $p_{0}$, see Fig. 3. The load displacement curve is depicted in Fig. 6. Furthermore the out-of-plane displacement in $z$-direction is plotted that shows the out-of-plane deformation of the cantilever beam due to the anisotropic material.

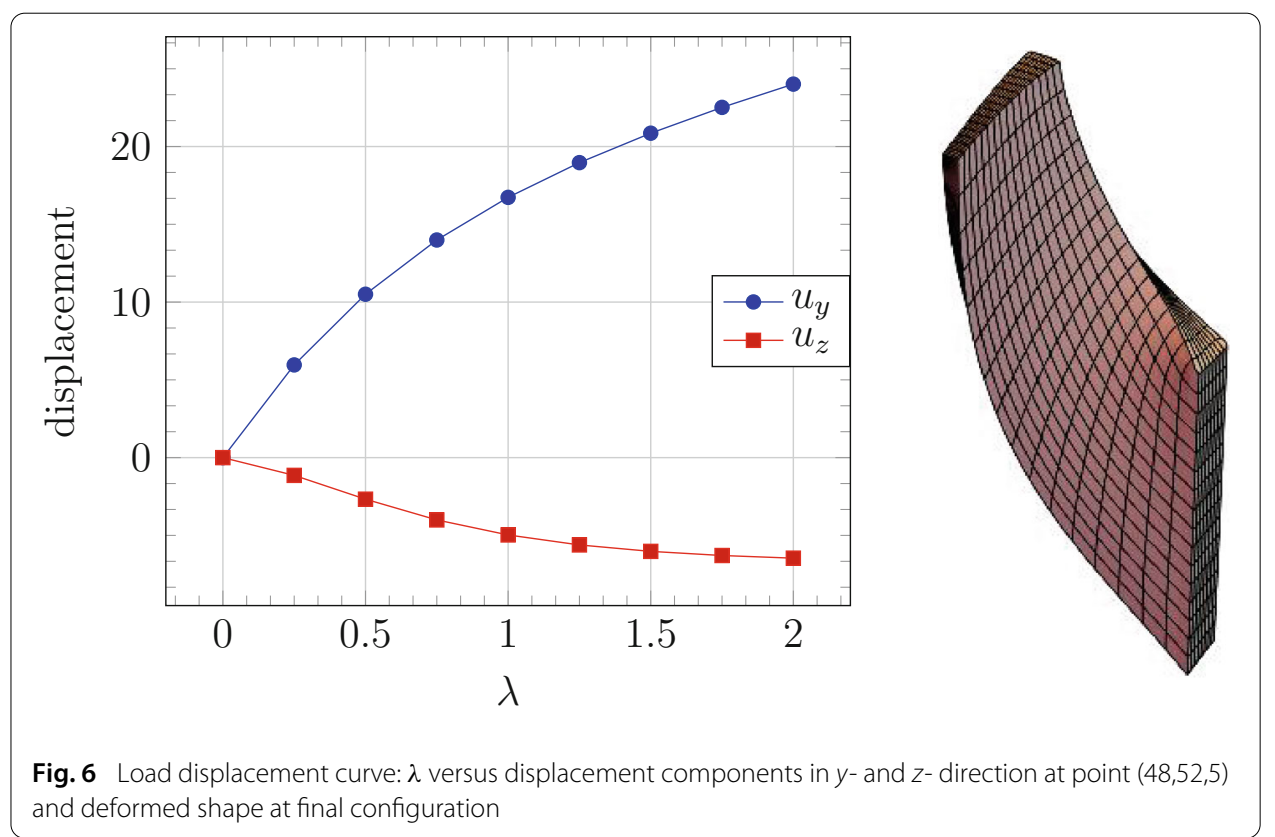


The deformed mesh on the right in Fig. 6 was computed with a mesh of $16 \times 16 \times 8$ elements which lead to a total number of 59058 degrees of freedoms. The deformation at the final configuration clearly depicts the twist in the deformed shape due to the anisotopic constraint at large deformations. The solution was computed with several load steps. In total eight load steps were applied for all discretizations reported in Fig. 7. The convergence behaviour was robust, six iterations per load step were needed for all discretizations to obtain convergence. In this solution procedure Newton type convergence was observed. When using the automatic load stepping scheme of AceFEM the total load can be applied in five load steps which reduces the total number of iterations to 33 and thus leads to reductions in computing time by a factor of around 1.5 .

A convergence study is performed for the fully constraint case, using the Lagrangian multiplier formulation (9). The element formulations $\mathrm{H} 2-\mathrm{A} 1$ and T2-A1 are compared. Figure 7 depicts the convergence of the vertical displacement at point $(48,60,0)$.

It can be observed that the hexahedral element performs slightly better for coarse meshes. Here one has to acknowledge that the coarsest mesh $(N=2)$ of the triangularization for the tetrahedral elements is not symmetric and thus will have a certain bias. Nevertheless the displacement for the coarsest mesh is close to the final result, being approximately only $5 \%$ off.

In order to show the dependency of the solution on the penalty or fiber stiffness parameter $C_{c}$ a series of computations were performed. The perturbed formulation (14) was used and a mesh division of $N=8$ selected.

Here it can be observed that the anisotropic constraint in direction of $\mathbf{a}$ is not enforced for a penalty parameter $C_{c} \leq 10$. Then there is an intermediate stage where the stiffness of the fiber changes the deformation state. This is related to parameters between $10 \leq C_{c} \leq 10^{5}$. Finally from $C_{c}>10^{5}$ on there is no further change, thus the parameter is sufficient to enforce the constraint. Additionally we note, that for $C_{c}>10^{7}$ the result is the same as for the pure Lagrangian multiplier formulation (9).

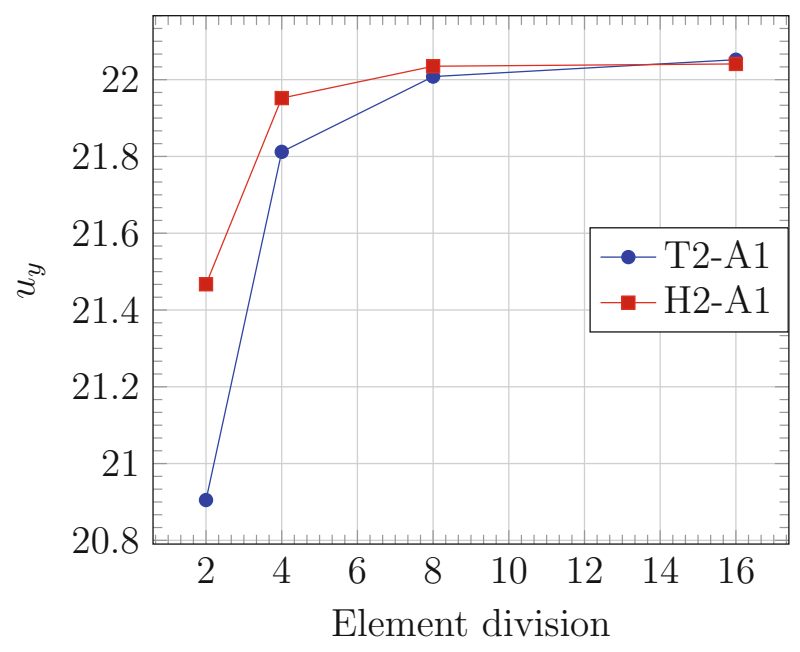

Fig. 7 Convergence study, constraint case 
A convergence study is now performed for the perturbed Lagrangian formulation, see (14). The results are compared with the penalty formulation (16) for a parameter of $C_{c}=10^{6}$. The results can be found in Fig. 9 .

It can be seen that the penalty formulation does not converge to the same solution as the perturbed Lagragnian formulation. Here a penalty parameter was used that is sufficient to fulfill the constraint, see Fig. 8. Thus it is clear from Fig. 9 that the penalty formulation locks. Furthermore it is interesting to observe that for a penalty parameter of $C_{c}>10^{7}$ the penalty method for the $\mathrm{H} 2$ as well as for the $\mathrm{T} 2$ element diverged while the perturbed Lagrangian formulations H2-A1-P and T2-A1-P are still robust.

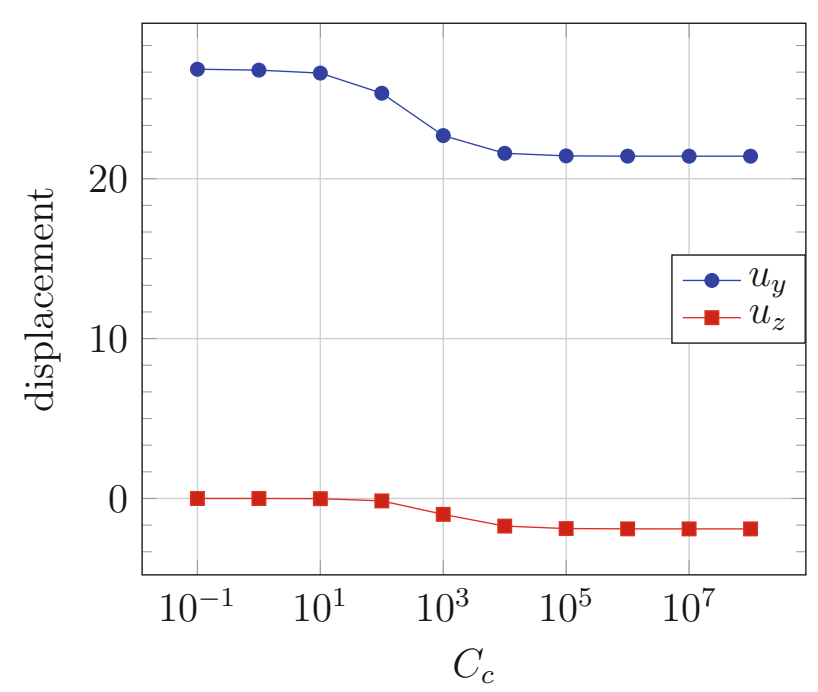

Fig. 8 Influence of the stiffness parameter $C_{c}$ on the displacement components in $y$-and $z$-direction at point $(48,60,5)$

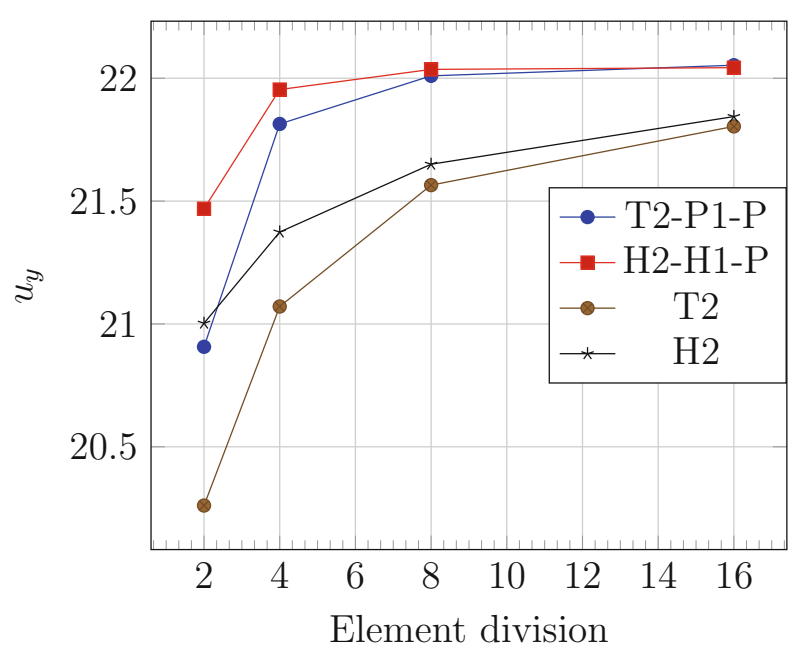

Fig. 9 Convergence study, penalty versus perturbed Lagrangian, $C_{p}=10^{6}$ 


\section{Shear deformation of a beam}

When a clamped beam is subjected to an end load then the beam will usually bend in the direction of the loading. In case that the axial movement is constraint the beam can only undergo shear deformations. The beam has a length of 40 , a height of 4 and a thickness of 2 (in dimensionless coordinates), see Fig. 10.

The constitutive data are provided for the Lame constants: $\mu=500$ and $\lambda=1000$. The direction of anisotropy is given by $\mathbf{a}=\{1,0,0\}$ which enforces the constraint in $x$ direction. The beam is clamped at the left end using the boundary conditions: $u_{x}=0$ for all nodes at $x=0, u_{y}=0$ for all nodes at $x=0$ and $y=0$ and $u_{z}=0$ for all nodes at $x=0$ and $z=0$. The beam is loaded by a constant traction of $p_{y}=5$ at the right end.

The pure shear state will now be enforced for the beam depicted on the left side of Fig. 10. The loading is such that a small strain state occurs. This leads to a deformed state that is reported on the right side of Fig. 10. Here the deformation is scaled by a factor of 20 . The displacement at the right side of the beam is $u_{y}=0.467$. Since the length of the beam is $L=40$ this displacement amounts to a shear deformation of $\gamma=u_{y} / L=0.01168$. This result can easily be checked using the classical beam theory. Here the shear deformation is

$$
\gamma_{B}=\frac{Q}{G \hat{A}}
$$

with $\hat{A}=\frac{5}{6} A, Q=p_{y} A, G=\mu$ and $A=2 \times 4=8$ it follows $\gamma_{B}=0,012$ which is very close to the computed value of $\gamma$.

For larger loads local buckling occurs. This is due to the high compressive stresses at the bottom of the beam. The load deflection curve in Fig. 11 depicts the nonlinear behaviour and the final deformation of the beam for a mesh with T2-A1-P elements. The deformed

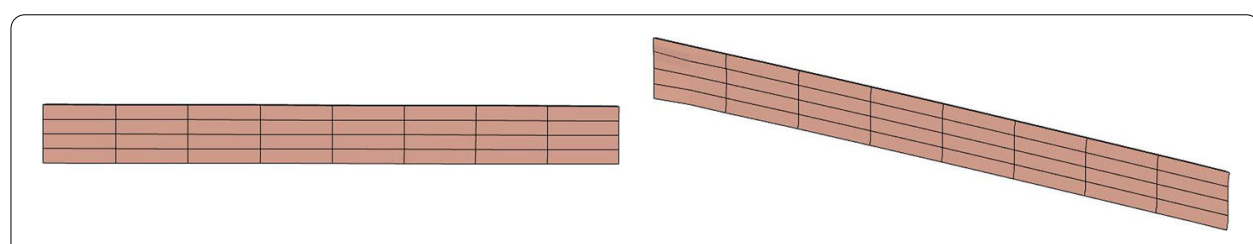

Fig. 10 Undeformed and deformaed configuration of the beam
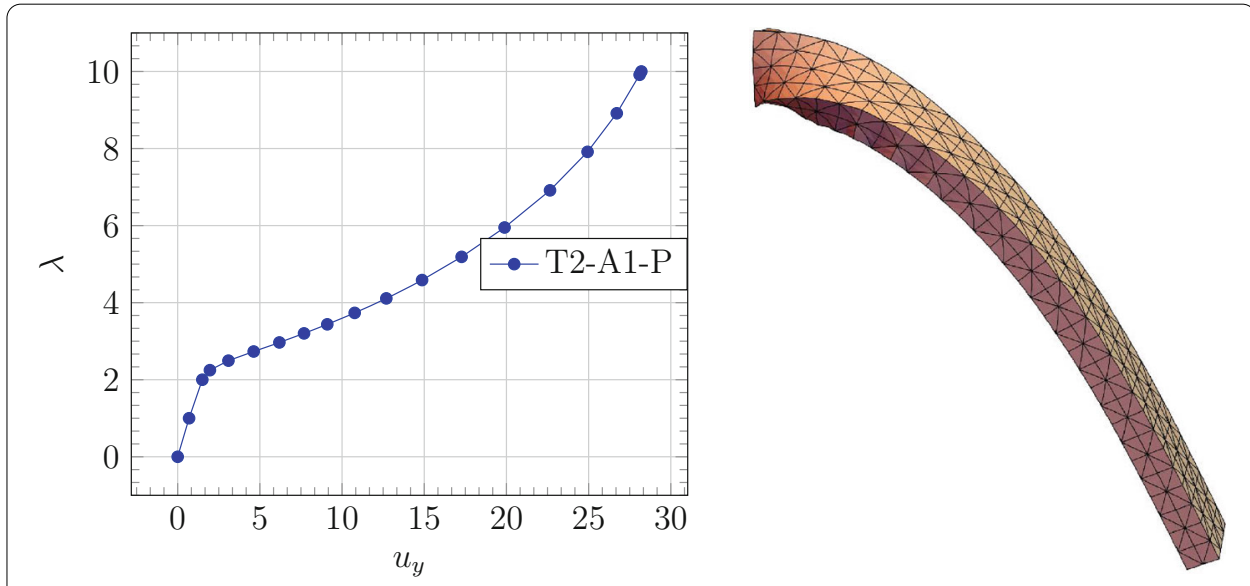

Fig. 11 Load deflection curve, T2-A1-P, $C_{C}=10^{6}$ and deformed beam 
configuration of the beam (no scaling) shows clearly near the clamping local buckles that in the end led to the large deflection of the beam. This is related to a bending torsion state which is triggerd by the local buckling.

It is clear that in reality an internal local buckling of the fibers will occur and thus the formulation (11) has to be applied. This leads then to a bending of the beam without local buckling, since fiber buckling due to compressive stresses is not present anymore. However, since the fibers in tension cannot extend, the deflection related to (11) is smaller than the deflection of a beam under bending without any constraints.

\section{Rolling up of a beam}

This example is related to a beam that is subjected to a prescribed stretch on its upper part. The problem is meshed with the quadratic hexahedra H2-A1-P and the quadratic tetrahedra T2-A1-P with $C_{c}=10^{8}$. A stretch of $\bar{\lambda}_{c}=1+\beta \epsilon_{c}$ with $\epsilon_{c}=-0.05$ is prescribed in the elements of the thin upper layer. The stretch is increased within 10 equal load increments $(\beta=1, \ldots, 10)$. The following boundary conditions are imposed at the left end of the beam in order to clamp the beam at this side: $u_{x}=0$ for all nodes at $x=0, u_{y}=0$ for all nodes at $x=0$ and $y=0$ and $u_{z}=0$ for all nodes at $x=0$ and $z=0$, see Fig. 12.

The selected finite element mesh is depicted on the left side of Fig. 13. The final state of the deformation is shown on the right side of Fig. 13. It is obtained for the load factor $\beta=10$.

It is clear that large strain states can be imposed by the formulation (12).

The displacements $u_{x}$ and $u_{y}$ are plotted versus the load factor $\beta$ in Fig. 14. It was obtained for a mesh with $N=8$ and the T2-A1-P element.

It can be conlcuded that the active enforcement of a given stretch using formulation (12) can be applied to generate arbitray deformation states depending on the selection of the direction vector a and the magnitude of the prescribed stretch $\bar{\lambda}_{c}$.

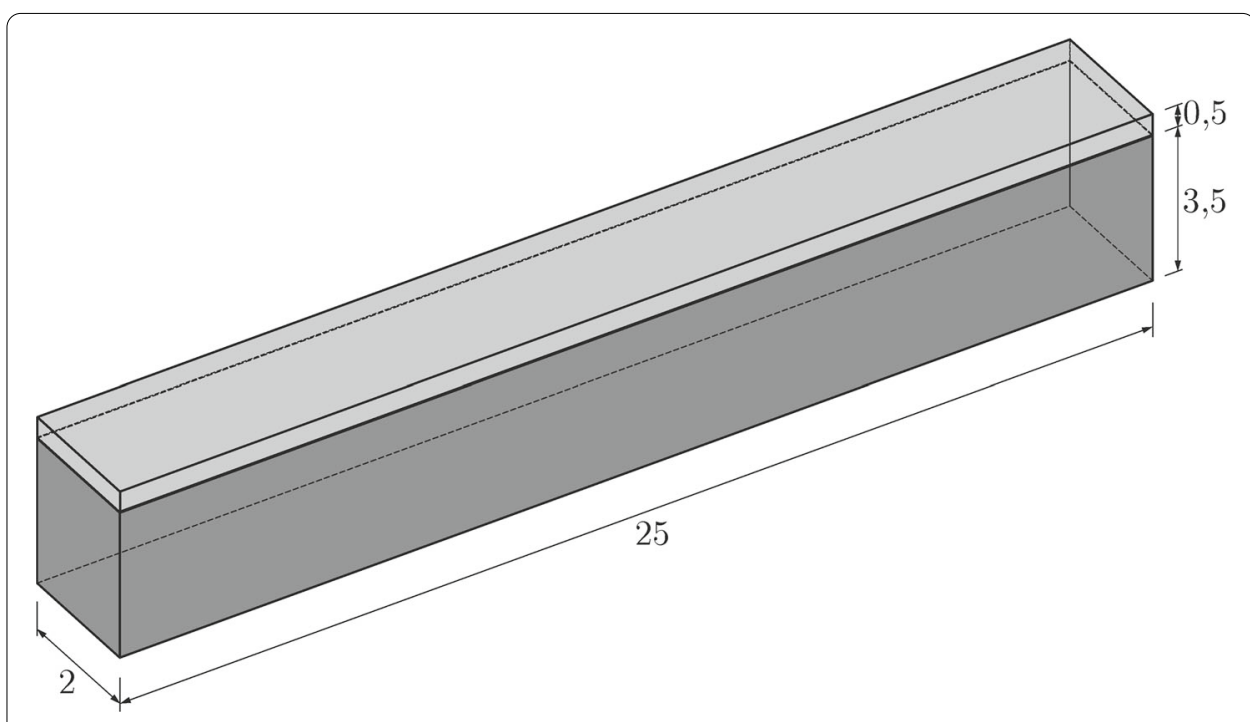

Fig. 12 Undeformed configuration of the beam for the H2-A1-P element 

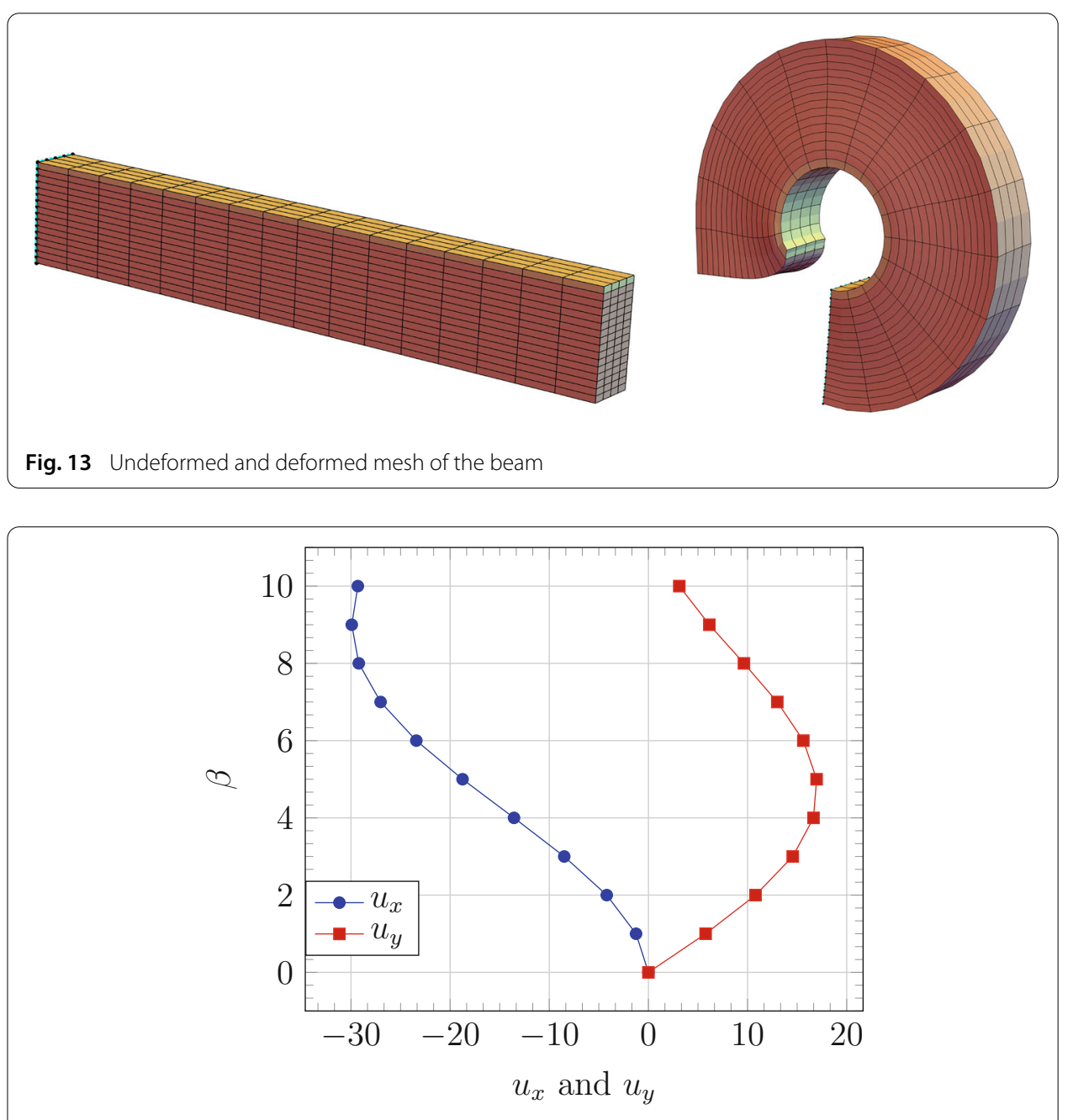

Fig. 14 Load deflection curve for the element T2-A1-P, $C_{C}=10^{8}$

\section{Bias extension test}

A problem where tension locking can occur is the tensile test where fibers are oriented in $\pm 45^{\circ}$ in the initial configuration. This bias-extension test was used in ten Thjie and Akkerman [26] and Hamila and Boisse [10] to investigate behaviour of standard finite element formulations and special interpolation techniques to avoid locking. The test is performed on a rectangular specimen, see Fig. 15 for the finite element mesh in the initial configuration. The length of the specimen is $L=300$, its width is $H=100$ and the thickness of the specimen is $T=10$. The specimen is clamped at both ends and pulled using a constant displacement $\bar{u}_{x}=65$. In order to obtain a two-dimensional plane stress state, as it was used in Hamila and Boisse [10], the displacements of all nodes where set to zero in thickness direction at $Z=0$. The material properties of the matrix material are described by the Lame constants $\lambda=1$ and $\mu=1$. The fiber stiffness is $C_{c}=4000$.

When the specimen is stretched from $L$ to $L+\bar{u}_{x}$ different in-plane shear zones occur, see Hamila and Boisse [10]. As depicted also in this paper, the computation using a standard element formulation, here a pure displacement formulation using T2 elements, yields a non physical deformation state, see left side of Fig. 16. On the other hand the new T2-A1 

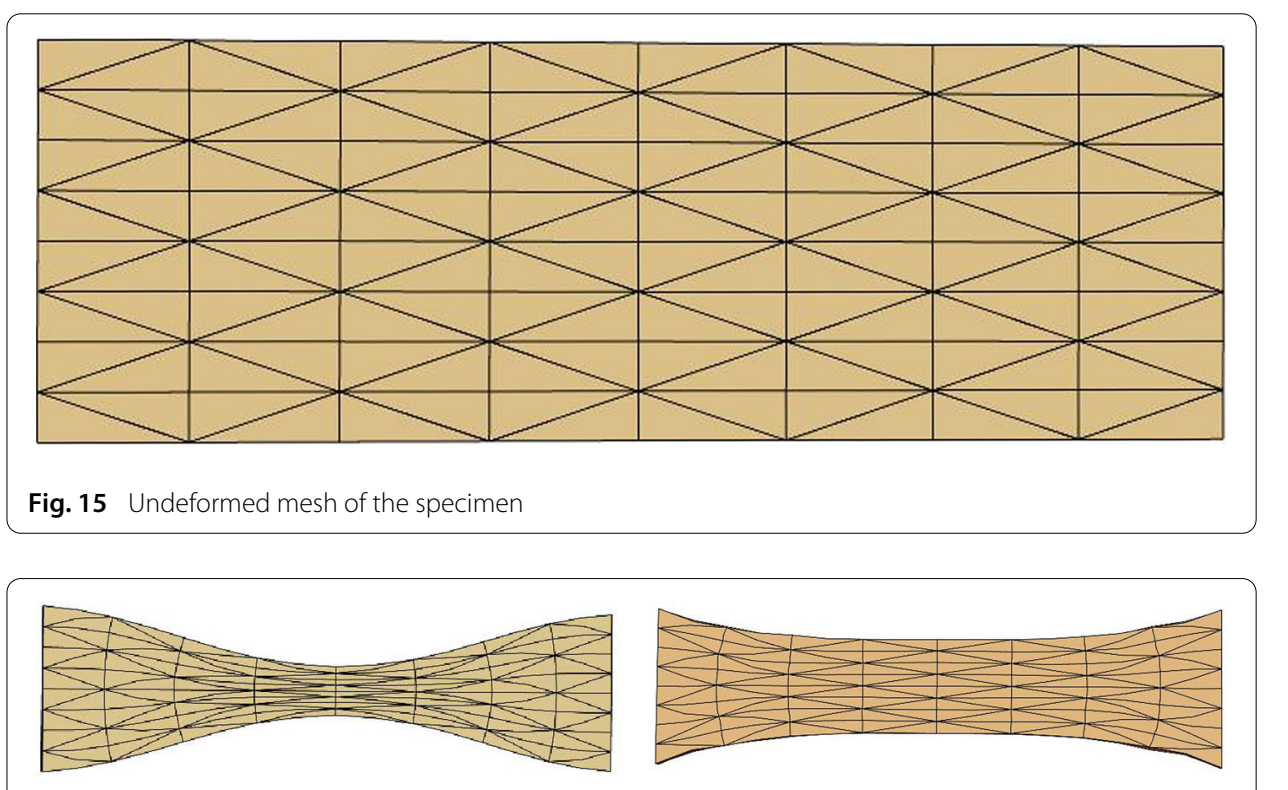

Fig. 16 Deformations states for T2 and T2-A1 element formulations

element yields even with a relatively coarse mesh a correct deformation pattern, which is depicted on the right side of Fig. 16 and has the same form as described in Hamila and Boisse [10]. The deformation of the finest mesh, see Fig. 17, actually shows also the different shear zones.

The plot in Fig. 18 shows the mesh convergence for the T2-A1 element formulation using $N=4,8,16,32$ and 64 elements per side. As can be seen the result is insensitive with respect to the mesh size. The deviation for $N=4$ is related to the fact that the mesh cannot model the different shear zones, see Fig. 17.

It is worth noting that the final displacement can be reached with the T2-A1 element in one single load step for all mesh sizes, while the T2 element needs about 25 load steps to reach the final configuration. Thus the new T2-A1 element is a lot more robust than the T2 element for such applications.

\section{Conclusions}

Finite elements for large strain anisotropic behaviour were developed in this paper. Special emphasis was put on a formulation that was able to enforce inextensible fiber extensions

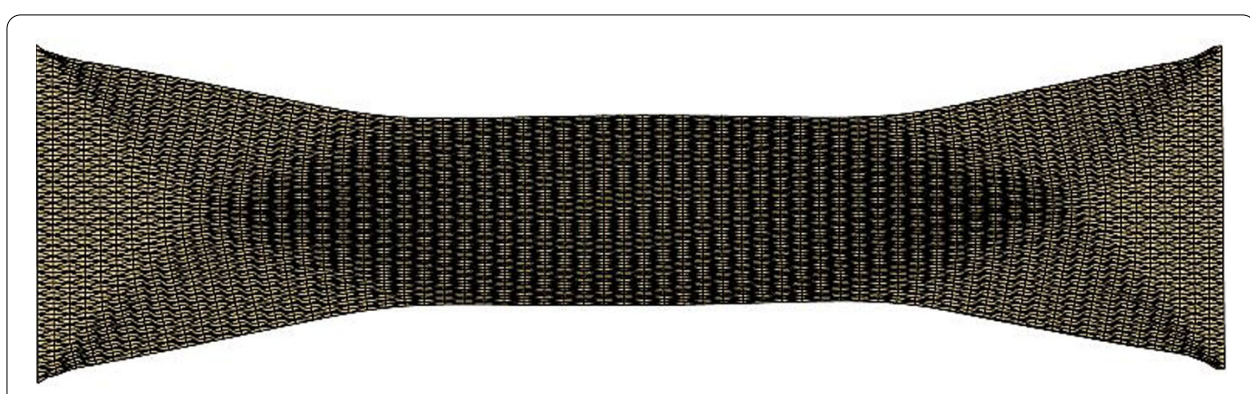

Fig. 17 Different shear zones obtained with a T2-A1 mesh of 40960 elements 


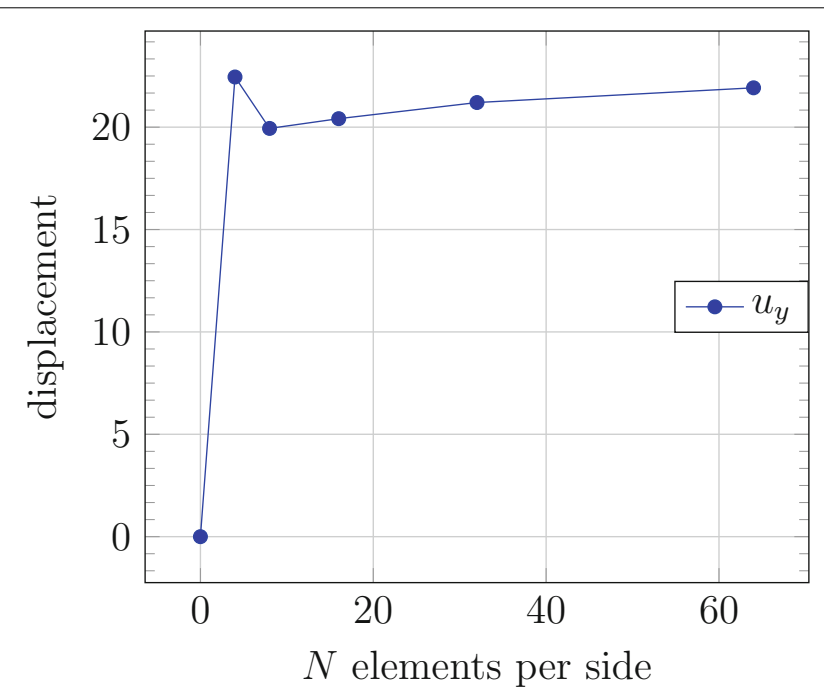

Fig. 18 Mesh convergence of the displacement $u_{y}$ in the midst of the specimen

for anisotropic materials exactly using a constraint formulation. This led to a Lagrange multiplier method with different ansatz spaces for the deformations and the Lagrangian multipliers (fiber stresses). The mixed approach shows a robust convergence behaviour and does not lock. A comparison with standard quadratic elements depicts the locking behaviour of these elements when the constraint was added via a penalty term. Furthermore the mixed approach led to a more robust behaviour in the iterative procedure needed to solve the associated nonlinear problems.

\section{Authors' contributions}

The theoretical derivations are joint work of PW, JS and FA. PW developed the AceGen Code. The Cook's membrane problem was developed by JS. The shear deformation problem of the beam stems from FA and the third example is due to PW. The final example was suggested by the reviewers. All authors read the approved the final manuscript.

\section{Author details}

${ }^{1}$ Institute for Continuum Mechanics, Leibniz Universität Hannover, Hanover, Germany, ${ }^{2}$ Institute for Mechanics, University of Duisburg-Essen, Essen, Germany, ${ }^{3}$ Department of Civil Engineering and Architecture, University of Pavia, Pavia, Italy.

\section{Acknowledgements}

The first and second author acknowledge the support of the "Deutsche Forschungsgemeinschaft" under contract of the SPP 1748, No. WR19/50-1 and SCHR570/23-1.

This contribution is dedicated to our friend and exceptional scientist Pierre Ladeveze on behalf of his 70th birthday.

\section{Competing interests}

The authors declare that they have no competing interests.

Received: 6 April 2016 Accepted: 13 July 2016

Published online: 11 August 2016

\section{References}

1. Auricchio F, de Veiga LB, Lovadina C, Reali A. A stability study of some mixed finite elements for large deformation elasticity problems. Comput Methods Appl Mech Eng. 2005;194:1075-92.

2. Auricchio F, da Velga LB, Lovadina C, Reali A, Taylor RL, Wriggers P. Approximation of incompressible large deformation elastic problems: some unresolved issues. Comput Mech. 2013;52:1153-67.

3. Babuska I. The finite element method with lagrangian multipliers. Numerische Mathematik. 1973;20(3):179-92.

4. Babuska I, Suri M. Locking effects in the finite element approximation of elasticity problems. Numerische Mathematik. 1992;62(1):439-63.

5. Bathe KJ. Finite element procedures. Upper Saddle River: Prentice Hall; 2006.

6. Belytschko T, Ong JSJ, Liu WK, Kennedy JM. Hourglass control in linear and nonlinear problems. Comput Methods Appl Mech Eng. 1984;43:251-76. 
7. Brezzi F. On the existence, uniqueness and approximation of saddle-point problems arising from lagrangian multipliers. Revue francaise d'automatique informatique recherche operationnelle Analyse numerique. 1974;8(2):129-51.

8. Brezzi F, Fortin M. Mixed and hybrid finite element methods. Berlin: Springer; 1991.

9. Chapelle D, Bathe KJ. The inf-sup test. Comput Struct. 1993;47:537-45.

10. Hamila N, Boisse P. Locking in simulation of composite reinforcement deformations. Analysis and treatment. Composites Part A. 2013;53:109-17.

11. Helfenstein J, Jabareen M, Mazza E, Govindjee S. On non-physical response in models for fiber-reinforced hyperelastic materials. Int J Solids Struct. 2010;47(16):2056-61.

12. Holzapfel G, Gasser T, Ogden R. A new constitutive framework for arterial wall mechanics and a comparative study of material models. J Elast Phys Sci Solids. 2000;61(1-3):1-48.

13. Hughes TRJ. The finite element method. Englewood Cliffs: Prentice Hall; 1987.

14. Korelc J. Automatic generation of finite-element code by simultaneous optimization of expressions. Theor Comput Sci. 1997;187:231-48.

15. Korelc J. Automatic generation of numerical codes with introduction to AceGen 4.0 symbolc code generator. http:// www.fgg.uni-lj.si/Symech. 2000.

16. Korelc J. Computational templates. http://www.fgg.uni-lj.si/Symech. 2016.

17. Reese $S$, Wriggers P. A new stabilization concept for finite elements in large deformation problems. Int J Numer Methods Eng. 2000;48:79-110.

18. Sansour C. On the physical assumptions underlying the volumetric-isochoric split and the case of anisotropy. Eur J Mech A/Solids. 2008;27(1):28-39.

19. Schröder J. Anisotropic polyconvex energies. In: Schröder J, editor. Polyconvex analysis, CISM. Wien: Springer; 2009 . p. 1-53.

20. Schröder J, Neff P. Invariant formulation of hyperelastic transverse isotropy based on polyconvex free energy functions. Int J Solids Struct. 2003;40(2):401-45.

21. Schröder J, Viebahn N, Balzani D, Wriggers P. A novel mixed finite element for finite anisotropic elasticity; the SKA-element-simplified kinematics for anisotropy. Submitted to computer methods in applied mechanics and engineering. 2016.

22. Simo JC, Armero F. Geometrically non-linear enhanced strain mixed methods and the method of incompatible modes. Int J Numer Methods Eng. 1992;33:1413-49.

23. Simo JC, Armero F, Taylor RL. Improved versions of assumed enhanced strain tri-linear elements for 3D finite deformation problems. Comput Methods Appl Mech Eng. 1993;110:359-86.

24. Simo JC, Rifai MS. A class of assumed strain methods and the method of incompatible modes. Int J Numer Methods Eng. 1990;29:1595-638.

25. Simo JC, Taylor RL, Pister KS. Variational and projection methods for the volume constraint in finite deformation elasto-plasticity. Comput Methods Appl Mech Eng. 1985;51:177-208.

26. ten Thjie RHW, Akkerman R. Solutions to intra-ply shear locking in finite element analyses of fibre reinforced materials. Composites Part A. 2008;39:1167-76.

27. Weiss JA, Maker BN, Govindjee S. Finite element implementation of incompressible, transversely isotropic hyperelasticity. Comput Methods Appl Mech Eng. 1996;135(1):107-28.

28. Wriggers P. Nonlinear finite elements. Berlin: Springer; 2008.

29. Zdunek A, Rachowicz W, Eriksson T. A novel computational formulation for nearly incompressible and nearly inextensible finite hyperelasticity. Comput Methods Appl Mech Eng. 2014;281:220-49.

30. Zienkiewicz OC, Taylor RL. The finite element method. 5th ed. Oxford: Butterworth-Heinemann; 2000.

31. Zienkiewicz OC, Taylor RL, Too JM. Reduced integration technique in general analysis of plates and shells. Int J Numer Methods Eng. 1971;3:275-90.

\section{Submit your manuscript to a SpringerOpen ${ }^{\circ}$ journal and benefit from:}

- Convenient online submission

- Rigorous peer review

- Immediate publication on acceptance

- Open access: articles freely available online

- High visibility within the field

- Retaining the copyright to your article

Submit your next manuscript at springeropen.com 